following parameters were monitored during the study: FVC\%, DLC\%, modified skin score (), activity index (EScSG), gastrointestinal tract symptoms, left ventricle ejection fraction, presence of diastolic ventricle dysfunction, PASP (Echo-CG), heart rhythm and conduction disoders (ECG), count of digital ulcers and necroses.

Results: MMF therapy led to a significant reduction of the mRSS $(7.5 \pm 6.9$ and $4.8 \pm 3.9, p=0.0006)$, EScSG (1.9 $\pm 1,5$ and $1.22 \pm 0.9, p=0.005)$, number of patients with heart conduction disorders $(13 / 29 \%$ and $5 / 11 \%, p=0.03)$.

The FVC improvement by $\geq 10 \%$ was documented in $6(13 \%)$ pts, and DLCO - in $3(7 \%)$; while worsening was observed in $4(9 \%)$, and $2(4 \%)$ cases, respectively. Mean FVC $(90.3 \pm 20.8$ and $92.2 \pm 21, \mathrm{p}=0.09)$, and DLCO $(52.2 \pm 17.4$ and 51.9 $\pm 17, p=0.86$ ) values did not change significantly.

CY therapy resulted in significant FVC increase $(80.5 \pm 20.1$ and $85.9 \pm 20.5$; $\mathrm{p}=0.034)$, reduction of EScSG $(2.8 \pm 2$ and $1.4 \pm 1.2, \mathrm{p}=0,0002)$ and $\mathrm{mRSS}(11,2$ $\pm 9,8$ and $7.9 \pm 6,8, p=0,009) . \geq 10 \%$ FVC increase was observed in $11(31 \%)$ pts, which was significantly more than in MMF group $(p=0.043)$. FVC loss was noticed in $2(5.6 \%)$ cases. The median FVC increase was $5.4 \%(25$ th $\%=-0.6,75$ th $\%$ $=12.3) . \geq 10 \%$ DLCO improvement and worsening was observed in $2(6.7 \%)$ pts. The mean DLCO values $(53,5 \pm 16,2$ and $54.4 \pm 15,5)$ did not change significantly. The other parameters monitored did not show significant deviations during the observation period.

Drug tolerability was better in MMF group: the rate of adverse drug reactions was significantly lower in MMF group (12/27\%), compared to CY group (19/53\%), $\mathrm{p}=0.03$.

Conclusions: Both drugs effectively reduced mRSS and EScSG in SSc patients. However, CY more often led to a clinically significant increase in FVC, in contrast to MMF, mostly contributing to stabilisation of FVC. The obtained results justify a differentiated approach to SSc pharmacotherapy depending on disease severity. A CY induction therapy should be considered as appropriate practice in patients with more severe pulmonary disease. A MMF induction therapy should be considered in SSc patients with cardiomyopathy and mild pulmonary disease, with poor CY tolerability. In other cases, MMF should be used for maintenance therapy after induction with $\mathrm{CY}$.

Disclosure of Interest: None declared

DOI: 10.1136/annrheumdis-2018-eular.4445

\section{SAT0506 SSC IN OLDER AGE: FREQUENT AND WITH A DIFFERENT PHENOTYPE. DATA OF THE GERMAN NETWORK FOR SYSTEMIC SCLEROSIS}

P. Moinzadeh ${ }^{1}$, G. Riemekasten ${ }^{2}$, N. Blank ${ }^{3}$, J. Henes ${ }^{4}$, I. Koetter ${ }^{5}$, E. Siegert ${ }^{6}$, C. Pfeiffer ${ }^{7}$, G. Zeidler ${ }^{8}$, M. Schmalzing ${ }^{9}$, C. Guenther ${ }^{10}$, L. Susok ${ }^{11}$, M. Worm ${ }^{12}$ A. Kreuter ${ }^{13}$, C. Sunderkoetter ${ }^{14}$, U. Mueller-Ladner ${ }^{15}$, A. Juche ${ }^{16}$, E. Aberer ${ }^{17}$, T. Schmeiser ${ }^{18}$, T. Krieg ${ }^{1}$, K. Kuhr ${ }^{19}$, N. Hunzelmann ${ }^{1}$. 'Dermatology, University Hospital, Cologne; ${ }^{2}$ Rheumatology, University Medical Center UKSH, Luebeck; ${ }^{3}$ Rheumatology, University Hospital, Heidelberg; ${ }^{4}$ Rheumatology, University Hospital, Tuebingen; ${ }^{5}$ Rheumatology, Asklepios Klinik Altona, Hamburg; ${ }^{6}$ Rheumatology, Charite Universitätsmedizin, Berlin; ${ }^{7}$ Dermatology, University Medical Center, Ulm; ${ }^{8}$ Rheumatology, Johanniter-Krankenhaus im Fläming, Treuenbrietzen; ${ }^{9}$ Rheumatology, University Hospital, Wuerzburg; ${ }^{10}$ Dermatology, University Hospital Carl Gustav Carus, Dresden; ${ }^{11}$ Dermatology, St. Josef Hospital, Bochum; ${ }^{12}$ Dermatology, Charite Universitätsmedizin, Berlin; ${ }^{13}$ Dermatology, HELIOS St. Elisabeth Klinik, Oberhausen; ${ }^{14}$ Dermatology, University Hospital, Muenster, ${ }^{15}$ Rheumatology, Kerckhoff Clinic, Bad Nauheim; ${ }^{16}$ Rheumatology, Immanuel Krankenhaus Berlin-Buch, Berlin, Germany, ${ }^{17}$ Dermatology, University Hospital, Graz, Austria; ${ }^{18}$ Rheumatology, St. Josef Hospital, Wuppertal; ${ }^{19}$ IMSB, University of Cologne, Cologne, Germany

Background: Systemic sclerosis (SSc) is a very heterogeneous multisystem connective tissue disease. The majority of affected patients develop initial clinical symptoms between the age of 30 to 50 years. It is not known whether an ageing population affects the clinical phenotype of SSc.

Objectives: To investigate the relationship of the age at disease onset and clinical characteristics in SSc patients using the registry of the German Network for Systemic Scleroderma.

Methods: Clinical data of the patient registry, currently including 4021 patients, were evaluated. Three age ranges at disease onset $(<40$ years, $40-60$ years, and $>60$ years) were correlated with clinical characteristics.

Results: Among all SSc patients, $27 \%$ of patients developed first non-raynaud symptoms at the age $<40$ years, while $44 \%$ developed SSc between $40-60$ years, and $29 \%$ were older than 60 years of age. In particular, SSc patients with disease onset $>60$ years developed significantly $(p<0.001)$ more often the IcSSc subtype (71.1\%), anti-centromere antibodies (45.8\%), had a significantly lower modified Rodnan Skin Score (mRSS) (7.8 \pm 8.1$)$, more often pulmonary hypertension $(\mathrm{PH})$ $(17.4 \%)$, a significantly lower mean DLCO level $(69.7 \% \pm 21.5)$ and less often digital ulcerations (20.2\%). However, a significant difference for lung fibrosis, heart or kidney involvement could not be observed. The more progressive subsets (dcSSc and SSc overlap syndromes) are found significantly more often at younger ages $(\mathrm{p}<0.001)$.

Conclusions: In this registry, nearly one third of patients developed SSc at an age above 60 years. These are mostly of the limited cutanous subtype with frequent $\mathrm{PH}$. These findings have an important influence on recommendations on diagnosis and theray of SSC.

Disclosure of Interest: None declared

DOI: 10.1136/annrheumdis-2018-eular.5214

\section{SAT0507 IMPLANTABLE LOOP RECORDER CAN SCREEN FOR INCIDENTAL SIGNIFICANT ARRHYTHMIAS IN SCLERODERMA, WITH CARDIAC MRI ECV AND TROPONIN BIOMARKER, USEFUL FOR RISK STRATIFICATION}

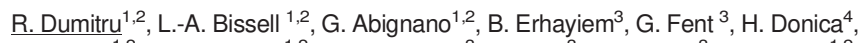
A. Burska ${ }^{1,2}$, F. Del Galdo ${ }^{1,2}$, J. Greenwood $^{3}$, S. Plein ${ }^{3}$, L. Graham ${ }^{3}$, M.H. Buch ${ }^{1,2}$. ${ }^{1}$ Leeds Institute of Rheumatic and Musculoskeletal Medicine, University of Leeds; ${ }^{2}$ NIHR Biomedical Research Centre; ${ }^{3}$ Leeds Institute of Cardiovascular and Metabolic Medicine, University of Leeds, LEEDS, UK; ${ }^{4}$ Department of Biochemical Diagnostics, University of Poland, Lublin, Poland

Background: Cardiac involvement and in particular conduction abnormalities represent a significant cause of morbidity and mortality in patients with systemic sclerosis (SSc). No studies assessed the value of implantable loop recorder (ILR) for early detection of arrhythmias in asymptomatic patients with SSc; or whether cardiac magnetic resonance (CMR) features associate with arrhythmias.

Objectives: To assess the prevalence of conduction abnormalities over a 3 year period using an ILR (REVEAL) and evaluate relationship with disease phenotype, cardiac biomarkers and CMR in SSc patients.

Methods: 20 patients(pts) with ACR/EULAR criteria for SSc, with no history of cardiovascular $(\mathrm{CV})$ disease and $\leq 1 \mathrm{CV}$ risk factor had $3 \mathrm{~T}$ CMR with late gadolinium enhancement (LGE) and T1 mapping for ECV quantification. An ILR was then inserted, for 3 years follow-up. ILR data were downloaded every 3 months. Serum cardiac biomarkers were also measured at the initial visit.

Results: 19 pts had available ILR data; 12 (63\%) females, median (SD) age 53 $\left(,^{12} 6(32 \%)\right.$ diffuse SSc(dcSSc), 6 (32\%) ACA+, 4 (21\%) Scl70+, $8(42 \%)$ history of interstitial lung disease (ILD) and 7 (36\%) history of digital ulcers (DU). 14/19 had any ILR abnormalities, 8/14 significant arrhythmias: 1 complete heart block; 2 non-sustained ventricular tachycardia (NSVT), and 5 atrial arrhythmias (1 atrial flutter, $1 \mathrm{AF}, 1 \mathrm{SVT}, 1 \mathrm{AF}$ and SVT and $1 \mathrm{AF}$ followed by atrial flutter and SVT). Of these 8 pts, 4 had dcSSc, 2 Scl70+, 4 ACA+, 3 with ILD and 3 DU history. All 3 patients with severe arrhythmias (NSVT/CHB) were dcSSc, $2 \mathrm{Scl} 70+, 2$ males. Management comprised 1 permanent pacemaker implantation, 3 antiarrhythmic treatment, 1 anticoagulation. 15 pts had CMR. The 8 pts with significant arrhythmia appeared to have higher ECV, LV mass, and LVEF\%(table 1). LGE was observed in 1 (NSVT), of a total of $5 / 15$ with LGE. HsTnl was considerably higher in those with significant arrhythmias[(mean diff.(95\% Cl)117 (-10, 245)].NTproBNP [(mean diff. $(95 \% \mathrm{Cl}) 92(-30,214)]$ also appeared greater in those with significant arrhythmias. There was no difference in CK levels between the two groups.

\begin{tabular}{|c|c|c|c|}
\hline CMR baseline & $\begin{array}{c}\text { ILR significant } \\
\text { arrhythmias, all years } \\
n=6 \\
\text { Mean(SD) }\end{array}$ & $\begin{array}{l}\text { ILR no significant } \\
\text { arrhythmias } \\
n=9 \\
\text { Mean(SD) }\end{array}$ & $\begin{array}{c}\text { Mean difference } \\
{[95 \% \mathrm{Cl}]}\end{array}$ \\
\hline ECV\% & $32\left(,^{2} n=5\right.$ & $29\left(,{ }^{4} n=9\right.$ & $2[-2,6]$ \\
\hline $\begin{array}{l}\text { LVEDV/BSA (ml/ } \\
\left.\mathrm{m}^{2}\right)\end{array}$ & $84\left(^{16}\right.$ & $83\left(^{20}\right.$ & $1[-21,22]$ \\
\hline LVEF\% & $62\left(^{4}\right.$ & $59\left(^{5}\right.$ & $2\left[{ }^{3-8}\right]$ \\
\hline $\begin{array}{l}\mathrm{LV} \text { mass/BSA(g/ } \\
\left.\mathrm{m}^{2}\right)\end{array}$ & $46 r^{7}$ & $44\left({ }^{14}\right.$ & $2[-10,15]$ \\
\hline $\operatorname{LVSV} / \mathrm{BSA}\left(\mathrm{ml} / \mathrm{m}^{2}\right)$ & $51\left(^{8}\right.$ & $49\left({ }^{11}\right.$ & $2[-9,13]$ \\
\hline $\begin{array}{l}\mathrm{LVESV} / \mathrm{BSA}(\mathrm{ml} / \\
\left.\mathrm{m}^{2}\right)\end{array}$ & $33\left(^{9}\right.$ & $34\left(^{10}\right.$ & $-2[-13,9]$ \\
\hline $\begin{array}{l}\text { Distensibility } \\
\left(10-{ }^{3} \mathrm{mmHg}^{-1}\right)\end{array}$ & $5(4)$ & $4(2)$ & $0.4[-3,4]$ \\
\hline Torsion $^{\circ}$ & $12\left(,{ }^{6} n=5\right.$ & $14\left(,{ }^{5} n=8\right.$ & $-2[-8,5]$ \\
\hline LGE & N 1/5 & N 4/9 & \\
\hline
\end{tabular}

Conclusions: This pilot study demonstrates the ability of ILR to detect life-threatening arrhythmia in asymptomatic SSc pt. The data suggest CMR ECV(but not LGE) and cardiac biomarkers, in particular hsTnl (indicating subclinical myocardial injury) may be able to identify at risk pts that would benefit from ILR screening Future studies can inform a risk model, and provide insight into the pathogenesis of SSc associated arrhythmias.

Disclosure of Interest: None declared

DOI: 10.1136/annrheumdis-2018-eular.7071 


\section{SAT0508 \\ ACE-INHIBITORS IN ARTERIAL HYPERTENSION IN SSC PATIENTS DISPLAY A RISK FACTOR FOR SCLERODERMA RENAL CRISIS - A EUSTAR ANALYSIS}

S. Adler ${ }^{1}$, P.A. Varisco ${ }^{2}$, O. Distler ${ }^{3}$, L. Buetikofer ${ }^{4}$, O. Kowal-Bielecka ${ }^{5}$, Y. Allanore ${ }^{6}$, G. Riemekasten ${ }^{7}$, P.M. Villiger ${ }^{8} .{ }^{1}$ Rheumatology, University Hospital Bern; ${ }^{2}$ Regional Medical Service, Bern; ${ }^{3}$ University Hospital Zurich, Zurich; ${ }^{4}$ Clinical Trials Unit, Bern, Switzerland; ${ }^{5}$ Warsaw Medical University, Warsaw, Poland; ${ }^{6}$ Cochin Hospital, Paris, France; ${ }^{7}$ University Hospital Schleswig-Holstein, Luebeck, Germany; ${ }^{8}$ University Hospital Bern, Bern, Switzerland

Background: Scleroderma renal crisis (SRC) represents a rare but life-threatening manifestation in systemic sclerosis (SSC). Survival remains poor despite therapeutic use of ACE inhibitors (ACEi). Factors influencing the risk of SRC are not well characterised. In particular, ACEi are discussed as promoting but also as protecting SRC.

Objectives: To investigate the effect of ACEi on SRC incidence in prospectively collected data of a cohort of SSc patients.

Methods: EUSTAR database analysis with focus on arterial hypertension, antihypertensive medication and glucocorticoids. Subgroup analysis of a dataset with defined documentation of medication from January 2009 until November 2017.

Results: Out of 14'524 patients in the database we identified 7'648 patients with at least one follow-up after 2009. 102 patients developed SRC in 27'450 personyears (py), representing an incidence of $3.72(3.06-4.51)$ per 1'000 py. In a multivariable time-to-event analysis adjusted for age, sex, disease severity and onset, arterial hypertension, tendon friction rubs, SCL70 and ACA positivity, 78 of 6083 patients developed SRC. Herein, use of ACEi displayed an increased risk for the development of SRC with a hazard ratio (HR) of 2.07 (95\% confidence interval (Cl):1.28-3.36). Calcium channel blockers (CCB), angiotensin receptor blockers, endothelin receptor antagonists and glucocorticoids did not influence SRC incidence. Medication strategies were not altered after renal crisis. Cumulative mortality 5 years after renal crisis was $18.6 \%(95 \% \mathrm{Cl}: 13.0 \%-26.3 \%)$.
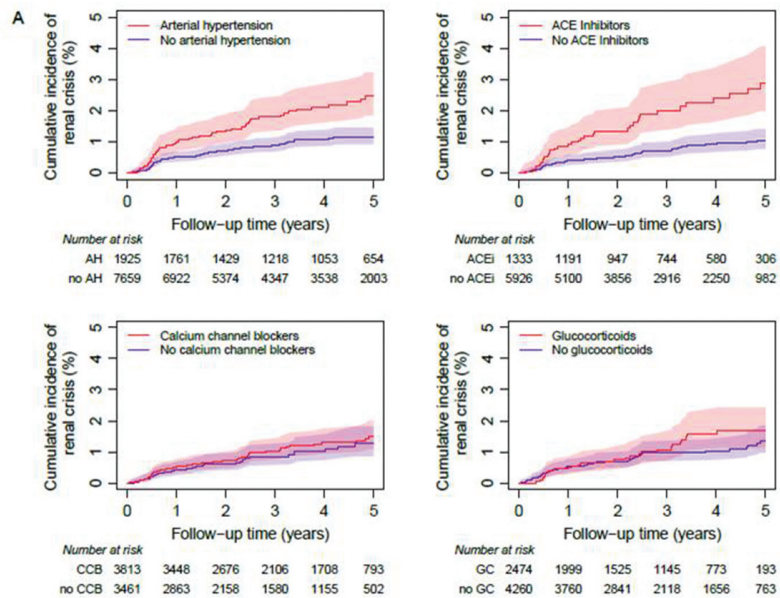

Figure (A) Cumulative incidence plots for scleroderma renal crisis (SRC) depending on whether patients have arterial hypertension (AH), or are treated with ACE inhibitors (ACE), Calcium channel blockers (CCB) or Glucocorticoids (GC).

Conclusions: This EUSTAR analysis supports the notion that ACEi should be avoided in arterial hypertension in SSc patients. Alternative antihypertensive drugs as CCB might be preferred.

\section{REFERENCES:}

[1] Hudson M, Baron M, Tatibouet S, Furst DE, Khanna D, International Scleroderma Renal Crisis Study I. Exposure to ACE inhibitors prior to the onset of scleroderma renal crisis-results from the International Scleroderma Renal Crisis Survey. Seminars in arthritis and rheumatism 2014;43(5):66672.

[2] Guillevin L, Berezne A, Seror R, Teixeira L, Pourrat J, Mahr A, et al. Scleroderma renal crisis: a retrospective multicentre study on 91 patients and 427 controls. Rheumatology (Oxford) 2012;51(3):460-7.

[3] Mouthon L, Bussone G, Berezne A, Noel LH, Guillevin L. Scleroderma renal crisis. The Journal of rheumatology. 2014;41(6):1040-8.
[4] Shanmugam VK, Steen VD. Renal disease in scleroderma: an update on evaluation, risk stratification, pathogenesis and management. Current opinion in rheumatology 2012;24(6):669-76.

Acknowledgements: We acknowledge the work of all participating EUSTAR centres for contributing to this work.

Disclosure of Interest: None declared

DOI: 10.1136/annrheumdis-2018-eular.2384

\section{SAT0509 HOW MONEY SHOULD BE SPENT: PATIENTS PERSPECTIVES ON SCLERODERMA RESEARCH FUNDING}

S. Divani ${ }^{1}$, J. Patel ${ }^{2}$, R. Stratton ${ }^{3} .{ }^{1}$ University College London Medical School;

${ }^{2}$ Homerton University Hospital; ${ }^{3}$ Royal Free Hospital, London, UK

Background: Scleroderma (systemic sclerosis, SSc) is an autoimmune disease that can affect blood vessels, skin and various organs, causing Raynaud's phe nomenon, thickening of skin, gastro-oesophageal reflux and lung fibrosis. Despite remarkable improvements and innovations in management for SSc, leaders in the field continue to believe there is scope for more. ${ }^{1}$ Patients' opinions on future research into the condition and its management, can provide valuable insight on how to shape its prospects and improve outcomes, and how research funding should be best directed. ${ }^{2}$

Objectives: To determine if doctors and patients with scleroderma agree on where future scleroderma research should be focussed.

Methods: 25 patients with scleroderma and 10 doctors were given question naires that asked them to rank 12 issues in order of importance, with a view to directing future Scleroderma research and funding. The highest ranked issue was appointed a score of 12 , and the lowest ranked appointed a score of 1 . The mean scores were calculated, and compared for concordance using ANOVA testing. All patients had been admitted for elective lloprost infusions at Royal Free Hospital and varied in age and gender. The clinicians were of varied levels from FY1 consultant, and all worked in the Rheumatology team at Royal Free.

Results: Patients ranked the issue of "managing Raynaud's symptoms" as the most important, which is significantly different to how clinicians viewed it - patients gave it a ranking 9.8 compared to only 4.9 from clinicians $(p<0.005)$. However doctors placed more value on "avoiding organ involvement" (10.4 from clinicians and 7.67 from patients $(\mathrm{p}<0.005))$.

Patients and clinicians, however, agreed about the least important issues, scoring "medication side effects" 3.6 and 3.1 respectively, "social and psychological support" 3.7 and 4.0, and "family planning and genetics" 3.8 and 3.6. They also agreed that research should be targeted at finding "new treatments that stop disease progression" which scored highly in both groups (9.7 in patients, 10.5 in clinicians).

Conclusions: Patients appear to feel that Raynaud's symptoms are affecting their quality of life in a way that is not being appreciated as important by clinicians. This may be due to ineffective communication between them, perhaps due to the time constraints of appointments.

Contrastingly, doctors are more concerned about avoiding organ involvement compared to patients, perhaps as they are more aware that organ involvement has a much greater effect on prognosis. However, both patients and clinicians were equally committed to investigating mechanisms of this disease.

Overall, these findings between doctors and patients should not just be a point of focus for future funding and research. It can be used to identify where there are dif ferences in expectations during clinical encounters, and therefore allow us as clinicians to tailor our agenda to better meet the needs of our patients, which would likely improve the patient-doctor relationship, enhancing communication in clinic appointments.

\section{REFERENCES:}

[1] The Lancet. Systemic sclerosis: advances and prospects. The Lancet 2017;390(10103):1624

[2] Sumpton D, Thakkar V, O'Neill S, Singh-Grewal D, Craig J, Tong A. "It's Not Me, It's Not Really Me." Insights From Patients on Living With Systemic Sclerosis: An Interview Study. Arthritis Care \& Research 2017;69 (11):1733-1742.

Disclosure of Interest: None declared DOI: 10.1136/annrheumdis-2018-eular.1556 